\title{
La réélection de Lula et la Gauche au Brésil
}

\section{Adilar Cigolini, Francisco Mendonça et Irani dos Santos}

\section{OpenEdition \\ Journals}

\section{Édition électronique}

URL : http://journals.openedition.org/echogeo/852

DOI : $10.4000 /$ echogeo.852

ISSN : 1963-1197

\section{Éditeur}

Pôle de recherche pour l'organisation et la diffusion de l'information géographique (CNRS UMR 8586)

\section{Référence électronique}

Adilar Cigolini, Francisco Mendonça et Irani dos Santos, «La réélection de Lula et la Gauche au Brésil », EchoGéo [En ligne], Sur le Vif, mis en ligne le 21 juin 2007, consulté le 19 avril 2019. URL: http://journals.openedition.org/echogeo/852 ; DOI : 10.4000/echogeo.852

Ce document a été généré automatiquement le 19 avril 2019.

\section{(c) (i) (9)}

EchoGéo est mis à disposition selon les termes de la licence Creative Commons Attribution - Pas d'Utilisation Commerciale - Pas de Modification 4.0 International 


\title{
La réélection de Lula et la Gauche au Brésil
}

\author{
Adilar Cigolini, Francisco Mendonça et Irani dos Santos
}

1 Le président du Brésil, Luiz Inacio Lula da Silva (Lula), du parti des Travailleurs - PT - a été réélu à la fin de l'année 2006 pour un deuxième mandat. En 2002, quand il a été élu pour la première fois, ayant face à lui le candidat José Serra (PSDB - Parti de la Social Démocratie du Brésil), soutenu par le président d'alors Fernando Henrique Cardoso (PSDB), Lula a gagné avec approximativement $61 \%$ des votes valides. A cette occasion, il a pris des voix non seulement à son challenger qui était dans le gouvernement depuis huit ans, mais aussi à une importante coalition de partis de droite et de centre-droit, l'ensemble des conservateurs qui ont dominé historiquement la politique brésilienne. Il a également dû faire face aux medias qui ont beaucoup oeuvré pour que le candidat du gouvernement sorte vainqueur des élections.

2 En ce début de millénaire, l'élection de Lula et du PT pour gouverner le Brésil constitue une marque incontestable dans la construction de la démocratie et de la gauche pour la sphère politique de l'Amérique Latine et pour le pays en particulier. Il s'agit du premier homme politique dont les origines sont modestes et du premier parti de gauche à prendre le pouvoir depuis cinq cents ans d'histoire brésilienne. Pendant toute cette periode, le Brésil a été dirigé par des gouvernements consécutifs parmi lesquels ont figuré des monarques ou des militaires de tout accabit, des bourgeois ou des capitalistes et leurs représentants directs... et la plupart du temps, de manière autoritaire, dictatoriale et despotique. Tandis que dans d'autres pays latino-américains, les expériences de la gauche au pouvoir étaient déjà bien connues (Cuba, Chili, Nicaragua, Le Salvador, etc), au Brésil, la classe dominante et plusieurs branches conservatrices de la société ont utilisé de multiples stratégies pour empêcher la constitution d'un gouvernement populaire malgré les manifestations de plusieurs mouvements de gauche pendant pratiquement tout le $\mathrm{XX}^{\mathrm{e}}$ siècle.

Bien que le PT affichait plusieurs expériences réussies dans certains gouvernements locaux et au sein de mairies de grandes capitales régionales, le succès 2002 n'a pas été une 
tâche facile. Outre, un nécessaire processus de sensibilisation de la population et des confrontations entre les medias et les secteurs conservateurs et catholiques les plus organisés, il a fallu aussi instaurer des coalitions avec des groupes sociaux d'opposition. Dans la «Lettre aux Brésiliens », Lula s'engageait contractuellement vis-à-vis de la stabilité économique. Ces engagements dans le champ économique ont été récupérés par certains organes conservateurs du gouvernement.

Les difficultés se sont considérablement accumulées dès la première année de l'arrivée au pouvoir de Lula (2003). A ce moment-là, son gouvernement comptait plus de $70 \%$ d'approbation populaire. C'était le moment idéal pour appliquer des réformes de base et de conduire le pays vers l'objectif rêvé par la gauche. Mais, outre les contraintes économiques, deux événements spécifiques ont rendu impossible l'exécution d'une politique de la gauche au Brésil : le manque d'un vrai projet socialiste de la part du PT et un contexte international marqué par la Guerre des États-Unis contre l'Irak, moment où une grave crise géopolitique a remis en question l'appui venant de l'étranger pour les transformations qui s'avéraient nécessaires. Les électeurs historiques du PT et de Lula sont rapidement tombés dans un état de frustration, et ce sentiment s'est manifesté pendant toute la durée du premier mandat. Dans la pratique, peu de choses ont changé, notamment pour les classes moyennes et les classes supérieures. Néanmoins, la classe inférieure a obtenu des acquis significatifs qui ont contribué à l'évaluation positive du premier mandat. Malgré ce contexte défavorable, aggravé par la crise politique liée aux affaires de corruption du congrès national, le président Lula s'est représenté et a été élu.

$\mathrm{Au}$ premier tour de l'élection de 2006, Lula a obtenu approximativement $48 \%$ des suffrages valides, tandis que son adversaire, Geraldo Alckmin (PSBD) n'a fait qu'environ $41 \%$. Au second tour, Lula a gagné l'élection avec grosso modo $60 \%$ des suffrages, alors que sont adversaire n'en recueillait $39 \%$. Au premier tour des élections pour un nouveau mandat, en nombre absolu de voix, Lula a élargi sa base d'électeurs : en passant de 53 millions en 2002 à 58 millions en 2006.

6 Le résultat a été, d'une certain manière, surprenant, car le PT et le gouvernement luimême étaient très critiqués en raison des graves affaires de corruption qui impliquaient les principaux alliés de Lula. Son premier mandat a connu deux crises qui auraient pu compromettre sa réélection. La première crise se rapporte à la déception d'une partie de la gauche et de la population qui attendait des réformes plus profondes. Mais, une fois au pouvoir, Lula a maintenu la politique économique héritée de son prédécesseur et a fait quelques réformes, comme celle de la sécurité sociale qui a réduit les droits des travailleurs. Cette mesure a été fortement critiquée par certains secteurs de la société liés à des mouvements syndicaux, à des partis de gauche et même au PT. La seconde crise a été marquée par les scandales de corruption portant sur le détournement d'argent pour couvrir des dépenses de campagne des partis de base du gouvernement au congrès. Les médias et l'opposition ont été implacables et ont cherché par tous les moyens à impliquer le président et sa famille dans ces scandales.

7 Dans ce contexte, beaucoup d'analystes politiques ont émis de sérieux doutes quant à la possibilité du maintien de Lula au pouvoir. Le résultat électoral, néanmoins, a montré que le peuple brésilien n'a pas suivi ces analyses et les prévisions des médias. Lula a gagné dans la plupart des territoires brésiliens et a renforcé sa position dans toutes les régions du pays, élargissant son implantation dans les petites villes de l'intérieur où jusque-là le PT rencontrait des difficultés à s'imposer. Dans des zones comme l'Amazonie - région Nord du pays, où se situe la forêt Amazonienne, Lula a obtenu 87 \% des suffrages. Le Nord- 
Est, région la plus pauvre du Brésil, a voté massivement pour Lula (77 \% des votes). Alors, Lula a-t-il été élu par les pauvres ? En grande partie oui, surtout lorsque l'on constate que le plus grand nombre de suffrages du candidat de l'opposition se concentre justement dans les états les plus riches. Mais, même dans ces états, le vote en faveur de Lula a été significatif puisque les électeurs de classe moyenne ont aussi rejeté les propositions du candidat Geraldo Alckmin (PSBD), l'associant aux thèses néo-libérales et aux privatisations réalisées pendant le gouvernement du président Fernando Henrique Cardoso et ont donné leurs voix au PT. La carte représentant la répartition économique des électeurs est fondamentale pour comprendre la réélection de Lula : elle montre un lien proportionnel incontestable entre l'IDH (Indice de Développement Humain) et le nombre de voix octroyées à la droite. En revanche, Lula a remporté la victoire dans les secteurs ou l'IDH est plus bas.

Illustration 1 - Election 2006 et indice de développement humain

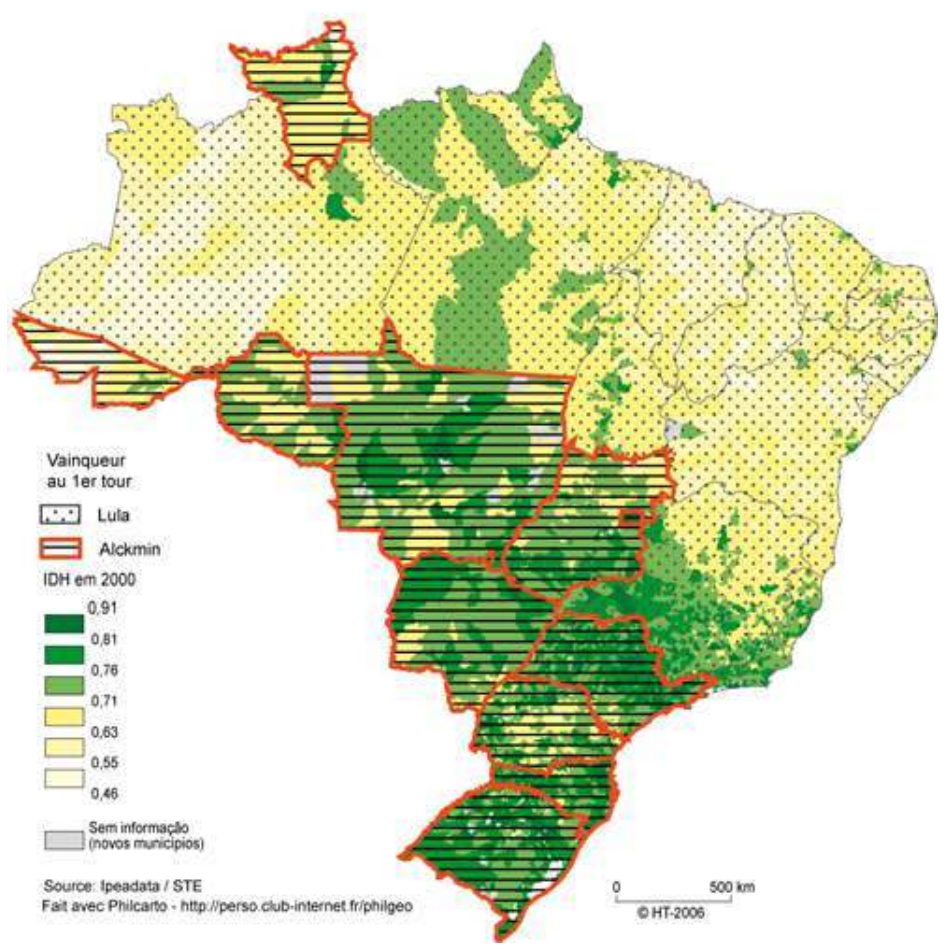

8 L'Etat brésilien a historiquement négligé les couches sociales défavorisées représentant des millions de Brésiliens noirs, indiens et métisses. Nous pensons que le gouvernement de Lula n'a pas utilisé suffisamment la force des mouvements syndicaux et des partis qui l'ont élu pour intensifier le processus de transformation sociale; toutefois, une des raisons essentielles qui l'ont porté à la présidence de la République, trouve son explication dans l'adhésion de la population pauvre à la politique du pays. Cela a non seulement entraîné de graves problèmes sociaux dans le quotidien des Brésiliens mais c'est aussi la première fois dans l'histoire que le peuple s'est trouvé au gouvernement, un gouvernement qui est favorable à la participation sociale comme forme de promotion de l'action de l'Etat. Le gouvernement a également accru la démocratie par l'organisation stratégique de conférences nationales ayant pour thème la santé, l'éducation, les villes et les droits humains. 
Par exemple, la Première Conférence Nationale sur l'Environnement a mobilisé environ 65000 personnes dans tout le pays pour discuter des directives et des actions à mener dans le secteur. Cette nouvelle dimension de la politique brésilienne change le rapport de force entre les partis, même ceux de droite, ce qui les amène à réviser leurs positions et approfondir leurs politiques sociales dans les régions qu'ils gouvernent.

Lors de la campagne du second tour en 2006, quand les programmes électoraux des deux candidats ont été plus précis, des milliers de militants et des mouvements sociaux ont soutenu Lula qui a accentué son discours à gauche et a promis un gouvernement meilleur que le premier. Il a clairement défendu les options politiques des pays voisins, renforcé le rôle du MERCOSUD et impliqué le Brésil dans d'importants organes internationaux de décision. Devant toutes les instances et dans toutes les situations, il s'est présenté comme un homme du peuple, ce qui l'a certainement aidé à gagner son second mandat. D'ailleurs, l' «homme du peuple » se montre sous un aspect symbolique très important, révélateur du ton charismatique entretenu entre le président et le peuple. Comme homme du peuple, migrant du Nord-Est du pays, travailleur et syndicaliste, Lula ne s'adresse pas aux Brésiliens avec un discours fait pour le peuple, il parle comme le peuple. Il y a donc une reconnaissance mutuelle entre Lula et les Brésiliens qui l'ont élu et il est par conséquent évident que le peuple se sente représenté par lui et s'identifie à lui.

11 Du point de vue économique, bien qu'ayant gardé des politiques conservatrices, Lula a réussi à stabiliser la situation économique, à contrôler l'inflation et à fortement réduire le niveau de risque de fuite des capitaux du Brésil (risque «Brésil» appelé aussi risque «Lula»). Concernant l'éducation, le gouvernement a mis en œuvre de nouvelles universités publiques et, par le biais d'un système d'exonération fiscale et de distribution de bourses d'études, il a réussi à créer approximativement 200000 nouvelles places gratuites dans l'enseignement supérieur privé. Il a mis sur pied de nouveaux ministères pour traiter de la question ethnique et de la lutte contre la violence envers les femmes. Il a mis en place des subventions et a élargi les programmes d'aide à l'agriculture familiale.

12 Au niveau social et dans le but de réduire la pauvreté, Lula a créé le programme « Faim zéro » et a fait un grand effort pour accomplir la promesse de campagne dans laquelle son gouvernement assurerait à tous, le droit à trois repas par jour. Il a instauré un ministère du développement social et du combat contre la faim et, en 2006, approximativement 10 millions des familles les plus pauvres du Brésil recevaient une aide financière par l'intermédiaire de politiques publiques permettant de garantir une alimentation correcte, de supprimer la pauvreté extrême et de promouvoir la citoyenneté chez plus pauvres. Outre les programmes d'intégration sociale, le salaire minimum a augmenté de plus de $25 \%$. Les classes D et $\mathrm{E}$, les plus pauvres, qui représentent $39 \%$ de la population, ont consommé $11 \%$ de plus pendant les quatre années du premier mandat. Tous ces facteurs ont contribué à ce que deux millions de familles, approximativement 8 millions de personnes, passent des classes $\mathrm{D}$ et $\mathrm{E}$ à la classe $\mathrm{C}$. Ceci est très important si l'on considère que le Brésil est l'un des pays les plus inégalitaires au monde. Le jour où il a été réélu, Lula a réaffirmé sa politique de transformation du Brésil en un pays plus juste ainsi que sa volonté de poursuivre cette voie. Lula a été réélu parce que les Brésiliens ont compris qu'il n'y a plus d'espace pour des politiques qui ne considéreraient pas le peuple comme élément central du processus de développement. Il a surtout été réélu parce qu'entre 2003 et 2006, il est parvenu à réduire le taux de pauvreté.

13 Mais le gouvernement de Lula s'inscrit dans la mouvance des autres pays de l'Amérique Latine, actuellement dirigés par des leaders originaires eux aussi des mouvements de 
gauche mais dont les politiques différent pourtant les unes des autres. Cette différenciation est due aux diverses étapes de l'évolution politique, sociale et économique de chaque pays. Au même moment où certains pariaient dans des processus quasirévolutionnaires de libération populaire du capital (Venezuela, Bolivie, Cuba), d'autres, comme c'est le cas du Brésil (mais aussi celui du Chili ou de l'Argentine, par exemple), cherchent à prendre des positions basées sur la négociation mais cependant, chacun avec leur spécificité, avec moins d'impact pour résoudre la situation sociale.

Pour prendre un exemple, en ce qui concerne les mesures de justice sociale pour une distribution plus équitable de la richesse, emblèmes historiques du mouvement de la gauche internationale, il n'existe pas une seule voie. Cela se remarque dans le contexte latino-americain comme un peu partout dans le monde. Outre, les spéculations relatives à la nécessaire interaction entre les pays latino-americains, il semble que c'est la gauche qui ait vraiment la meilleure solution pour en finir avec l'exclusion sociale produite par la concentration du capital. L'actuelle expérience brésilienne du gouvernement de Lula, en apportant dans une démarche positive des solutions aux problèmes, met en évidence qu'il est nécessaire d'aller chercher de nouvelles alternatives pour la gauche et pour toute la société.

15 N.B. : Compte tenu du nombre de signes alloués, il ne nous a pas été possible de prendre en compte la longue carrière de syndicaliste de Lula ni ses 3 tentatives d'accès au pouvoir en 1989, 1994 et 1998.

\section{RÉSUMÉS}

Contrairement aux prévisions, Lula fut massivement réélu en 2006 avec l'appui des populations les plus défavorisées pour lesquelles il a mis en œuvre des mesures sociales notamment contre la faim, la discrimination ethnique ou la violence envers les femmes. Dans son premier mandat, il a promu l'action de l'Etat en instaurant la démocratie participative sur les thèmes de la santé, l'éducation, l'aménagement, l'environnement ou les droits de l'Homme, à laquelle les classes moyennes ont aussi adhéré. Grâce à sa personnalité charismatique et à son origine modeste, le peuple a le sentiment d'être représenté et s'identifie à lui. Economiquement parlant, il a néanmoins maintenu les grandes orientations du gouvernement précédent et par le biais de la négociation, il a renforcé le rôle régional (MERCOSUD) et international du Brésil. Ceci montre qu'en Amérique Latine, les politiciens doivent désormais compter avec le peuple pour gouverner.

Contrary to the forecasts, Lula was massively re-elected in 2006 with the support of the most underprivileged populations for which it implemented social measures in particular against the hunger, ethnic discrimination or violence towards the women. In its first mandate, it promoted the action of the State by founding the participative democracy on the topics of health, education, installation, the environment or the human rights, which the middle class also appreciated. Thanks to his charismatic personality and his modest origin, the people feel represented and is identified with him. Economically speaking, it nevertheless maintained the main trends of the preceding government and by the means of the negotiation, it reinforced the 
international and regional role (MERCOSUD) of Brazil. This shows that in Latin America, the politicians must from now on take into account the people to govern.

\section{INDEX}

Keywords : Brazil, geopolitics, Latin America, Lula, public politics

Mots-clés : Amérique latine, Brésil, élection, géopolitique, politique publique

\section{AUTEURS}

\section{ADILAR CIGOLINI}

Adilar Cigolini est master science en géographie politique et professeur à l'Université Fédérale du Paraná - Curitiba / Brésil

Ses deux dernières publications:

Cigolini, Adilar Antonio. 2002. A fragmentação do território em municípios: um resgate histórico. Revista Paranaense de Geografia, Curitiba, v. 5, p. 55-66.

Cigolini, Adilar Antonio. 2002. Ocupação do Território e Fragmentação Territorial: A formação da Malha Municipal Paranaense. Revista Paranaense de Geografia, Curitiba, v. 7, p. 23-35.

\section{FRANCISCO MENDONÇA}

Francisco Mendonça est docteur et Phd en géographie et environnement et professeur titulaire à l’Université Fédérale du Paraná - Curitiba / Brésil

Ses deux dernières publications:

Mendonça, Francisco. Le Bresil: Enjeux et défis d'une "puissance annoncée". In: Mendonça, Francisco.; Bertrand, Fredric. (Org.). 2006. Grafigéo - Le Bresil : Geoplitique et environnement actuels. $n^{\circ} 30$ Paris, Prodig, p. 31-40.

Mendonça, Francisco. (Org.). 2004. Cidade, ambiente e desenvolvimento. Abordagem interdisciplinar de problemáticas socioambientais urbanas de Curitiba e RMC. Curitiba: Editora da UFPR Imprensa Oficial do Estado do Paraná, v. 1. 276 p.

\section{IRANI DOS SANTOS}

Irani dos Santos est Master Science en Géographie Des Resources Hydriques et professeur à 1 'Université Fédérale du Paraná - Curitiba / Brésil Deux dernières publications: Santos, Irani dos ; Cigolini, Adilar . Tudo o que existe é: considerações sobre a idéia de natureza em Milton Santos. 2007. In: Luiz Fernando Scheibe; Adriana Dorfman. (Org.). o Curso da Teoria: ensaios a partir d'A Natureza do Espaço. Florianópolis.

Santos, Irani dos ; Fill, Heinz Dieter ; Sugai, Martha R V B ; Buba, Homero ; Kishi, Regina Tiemy ; Marone, Eduardo ; Lautert, Luiz Fernando C. 2001. Hidrometria Aplicada. Curitiba: LACTEC. v. 1. 372 p. 